\title{
¿Por qué un dossier sobre publicaciones periódicas de comparatismo?
}

Adriana Crolla

Universidad Nacional del Litoral

A 22 años de la creación del Centro de Estudios Comparados y casi 20 años del inicio del trabajo de edición de esta revista ${ }^{1}$ se nos presentó como interesante proponer una mirada sobre las publicaciones periódicas que la acompañan en la constitución del campo. Para ello convocamos a los responsables de las que se editan en Argentina y en algunos espacios académicos del extranjero con los que el CEC ha establecido contacto.

Esta indagación de corte archivístico se nos presenta como potente pues permite, al modo de los informes periódicos que algunos espacios propician, que el lector pueda establecer líneas de recurrencias o particularidades que hagan visible el modo en que desde los ámbitos multiversos de las universidades, se generan y potencian acciones permanentes o continuadas que aceptan el desafío de medir paralelos y distancias desde el campo de referencia que a cada comparatista le cabe mejor. Aceptar el desafío del comparatismo donde no siempre existen tradiciones académicas fuertes, donde las subvenciones no siempre están a la mano y las tensiones e intenciones entorpecen y abortan, lograr sostener y compartir el saber desarrollado es un logro a celebrar. Sobre todo al permitir una revisitación conjunta de tradiciones plurales y la proyección hacia acciones futuras coordinadas y reflexivas sobre el motor de nuestras comparaciones.

Además de las respuestas que hemos recibido a nuestra convocatoria, es necesario destacar en el ámbito argentino, la existencia de la Asociación Argentina de Literatura Comparada (AALC), nacida en una mítica reunión realizada en Buenos Aires en septiembre de 1992 a instancias de los Prof. Nicolás Dornheim y de Jorge Dubatti. Creada formalmente en 1993, contó con la presencia y entusiasmo permanente de la Dra. Tania Franco Carvalhal de la Universidad de Porto Alegre (Brasil) y del Dr. Jean Bessière de la Universitè de la Sorbona de Paris, quienes jugaron un papel de relevancia para la determinación de sus perfiles constitutivos. A semejanza de otras similares de Brasil y Europa, se estipuló por estatuto la organización de reuniones periódicas bianuales, recayendo la sede en la afiliciación académica del Presidente. En estos momentos la preside el Prof. Gabriel Matelo de la Universidad de La Plata. La AALC brinda apoyo a toda actividad que promueva y amplíe el campo y ha organizado hasta la fecha 12 jornadas en diferentes universidades.

En relación al objetivo de este dossier, destacamos que cada grupo organizador edita para tal fin la Revista Confluencias, que se entrega a los participantes y donde se aglutinan las novedades y acciones realizadas en el período que media entre un encuentro y otro. Desde la creación de la AALC se editaron Confluen- 
cias I (Mendoza, 1993); II (Córdoba, 1994); III (Córdoba, 1996); IV (Tucumán, 1998); V (Buenos Aires, 2001); VI (Mendoza, 2007), VII Santa Fe (2009), VIII La Plata y IX Buenos Aires. El número X de la revista, que debía ser distribuida en último encuentro en la Universidad de Córdoba en 2017, no pudo ser editada y se distribuirá en 2019 en la sede de La Plata. Confluencias, como boletín informativo de la Asociación, da a conocer las actividades y proyectos de los distintos centros universitarios del país dedicados a la Literatura Comparada y la serie permite tomar conocimiento de los recorridos y las producciones más relevantes de las investigaciones que se realizan en el país, en los países de Latinoamérica y en Europa, tanto en los aspectos teóricos como en el estudio de las ficciones de distintas geografías abordados en perspectivas comparadas. La página web de la AALC (https://aaliteraturacomparada.wordpress.com/) ha destinado un espacio específico donde es posible consultar cada una de estas publicaciones.

\section{Nota}

${ }^{1}$ Si bien el primer número es de diciembre del 2002, la revista comenzó a pensarse y diseñarse en el año 1999, cuando la facultad asumió una política editorial para acompañar las acciones de sus centros de estudios. 